\title{
Simulation research of bionic image intensifier based on the retina structure of elephant-nose fish
}

\author{
Hongxia ZHANG ${ }^{1,2 *}$, Qingwen JIN ${ }^{1,2}$, Peng LI ${ }^{1,2}$, DAgong JIA ${ }^{1,2}$, Tiegen LIU ${ }^{1,2}$ \\ ${ }^{1}$ College of Precision Instrument and Opto-Electronics Engineering, Tianjin University, \\ Tianjin 300072, China \\ ${ }^{2}$ Key Laboratory of Optoelectronics Information Technical Science, EMC, \\ Tianjin 300072, China \\ *Corresponding author: hxzhang@tju.edu.cn
}

\begin{abstract}
Enhancing image brightness under low light conditions is crucial. The traditional night vision technology depends on the development of photoelectric materials and accurate image processing algorithms. Inspired by the structure of elephant-nose fish, we construct a bionic image intensifier (BII) model. The BII is a planar array based on the concentrating principle of the compound parabolic concentrator (CPC). This solution is based on pure optical design and independent. Through light tracing, the simulation results show that the brightness of the image is improved and good image uniformity is obtained. Quantitative analysis of the geometric concentration ratio (GCR) and the image uniformity is used to optimize CPC structure parameters. At the same time, we also discuss the influence of CPC structural parameters on image continuity, uniformity and geometric concentration ratio. The optimization results are as follows: the input port diameter is $50 \mu \mathrm{m}$, the output port diameter is $10 \mu \mathrm{m}$, the height is $90 \mu \mathrm{m}$, the GCR is 5.61 , and the image uniformity is $95.30 \%$. The comprehensive performance of the BII achieves the best.
\end{abstract}

Keywords: geometric optical design, micro-optical devices, image analysis, low-light night vision, bionic image intensifier.

\section{Introduction}

Human visual sense ability will drop dramatically when the illumination levels of the environment decrease at night. Human eyes have reduced visual acuity in conditions of low illumination because of the limitations of the structure of the human retina [1]. It is not only difficult to judge depth and distance, but it is also difficult to differentiate colors. The details of the image are obscured [2]. Improving the image brightness in conditions of low illumination is very beneficial to the extraction of visual information. And it plays a crucial role in many applications such as medicine, military, security, industry, astronomy, etc. 
At present, the main method of increasing the brightness is to use a low-light night vision instrument. The low-light night vision instrument mainly includes an imaging lens group (ILG), a low-light image intensifier (LLII) and a post-processing system, in which the LLII is a core component of the low-light night vision instrument [ $\underline{3}-\underline{5}]$. The working principle of the low-light night vision instrument is that the low-light is imaged at the focal plane of the image intensifier photocathode. The image intensifier performs photoelectric conversion, electronic imaging, and brightness enhancement on the object. A bright image is displayed on the screen of the image intensifier for observation by human [ $\left.\underline{5}_{-}^{-7}\right]$. Low-light night vision technology is particularly dependent on electronics, including on-chip multiplication gain technology, or highly photosensitive imaging sensors with emerging photoactive materials, as well as precise image processing algorithms. Therefore, if we want to further enhance the low-light night vision technology, it has certain limitations in terms of materials [ $\underline{8}-11]$.

However, the eyes of some animals that survive in low-light conditions have a unique structure and good adaptability. Animal eyes have an advantage over human eyes $[\underline{12}, \underline{13}]$. For example, the family Mormyridae, sometimes referred to as "elephant fish" or "elephant-nose fish", are freshwater fish in Africa. They live in muddy water, but they can still successfully complete predation. This is related to the unique retinal structure of their eyes, which is called the "grouped retina". The retina is composed of tiny cups, acting like parabolic mirrors. The cups are made of light reflecting proteins, funneling red light to areas of cone that have adapted to see only red light because of muddy water, intensifying its brightness 10 -fold, while the other wavelengths of light are absorbed [14-16]. The retinal structure of the elephant-nose fish eye shows excellent brightness enhancement.

In this paper, we have designed a bionic image intensifier (BII) inspired by the retinal structure of the creature elephant-nose fish eye. Based on the principle of pure optics, we use a planar array composed of compound parabolic concentrators (CPCs) to effectively improve the brightness of the image. Image quality and image brightness are simulated using ILG and BII. The simulation results show that under low illumination conditions the brightness of the image is improved and the good uniformity is ensured by adjusting the CPC structure parameters.

\section{Model description}

References [14] provide several real structures of the retina of the elephant-nose fish. The retina is observed with the electron microscope (EM). The sketch map of the retina is shown in Fig. 1. The retina is composed of tiny cups, acting like parabolic mirrors. The size of the tiny cup is on the scale of micron. Based on this biological structure, we build a mathematical model.

The mathematical model is a micrometer-scale CPC model according to the geometric structure of the reflector cup. The profile of CPC structure is shown in Fig. 2. $\mathrm{CPC}$ is composed of two parabolas. Parabola $\mathrm{A}$ and parabola $\mathrm{B}$ are symmetrical to each other. The focus of the parabola $\mathrm{A}$ is $F_{1}$ and the focus of the parabola $\mathrm{B}$ is $F_{2}$. Two 


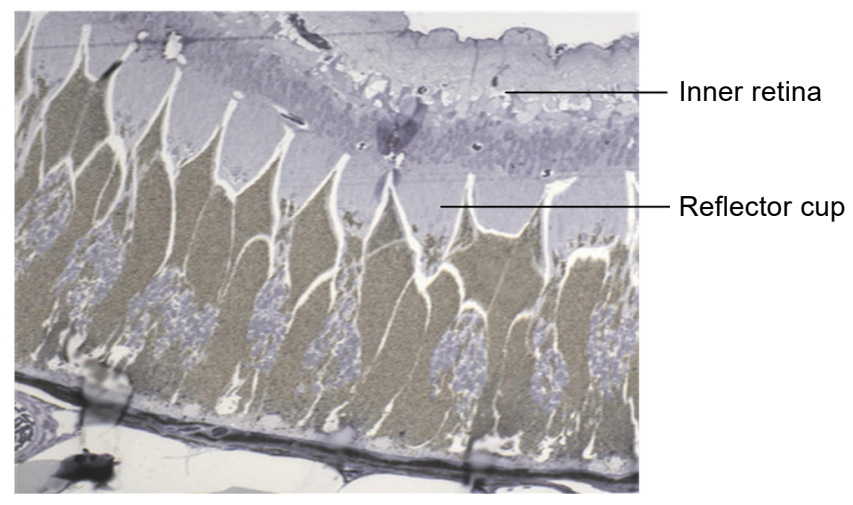

Fig. 1. The retina structure of the elephant-nose fish.

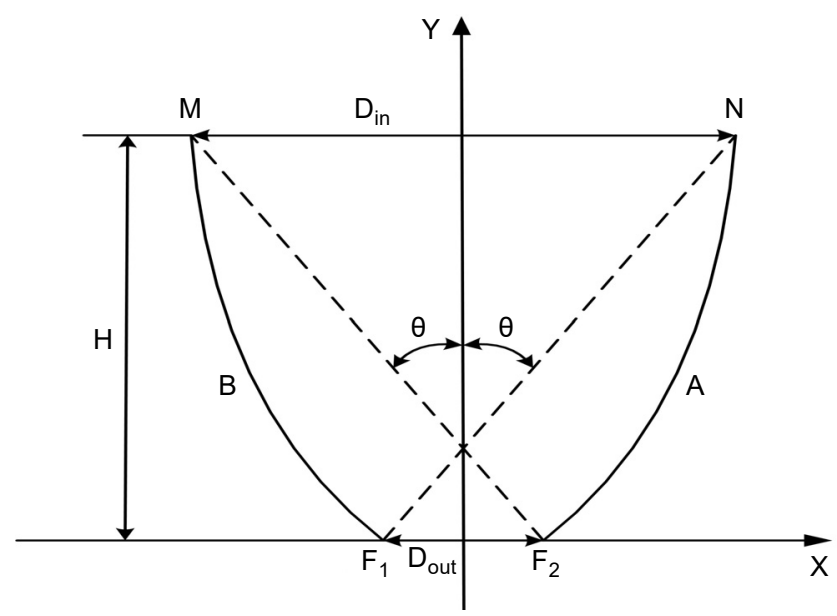

Fig. 2. The profile of CPC structure.

parabolas rotate $\theta$ degrees around their respective focal points. $D_{\text {in }}$ is the diameter of the input port of CPC. $D_{\text {out }}$ is the diameter of the output port of the CPC. $H$ is the height of the CPC.

The parameter equation [17- $\underline{19}]$ of $\mathrm{CPC}$ are:

$$
\begin{aligned}
& x=\left(4 f t^{2}-f\right) \sin \theta-4 f t \cos \theta+\frac{f}{1+\sin \theta} \\
& y=\left(4 f t^{2}-f\right) \cos \theta+4 f t \sin \theta
\end{aligned}
$$

In the equations, $t$ is a parameter variable which relates to $x$ and $y, f$ is the focal length of CPC, the expression for $f$ is

$$
f=\frac{D_{\text {out }}}{2}(1+\sin \theta)
$$



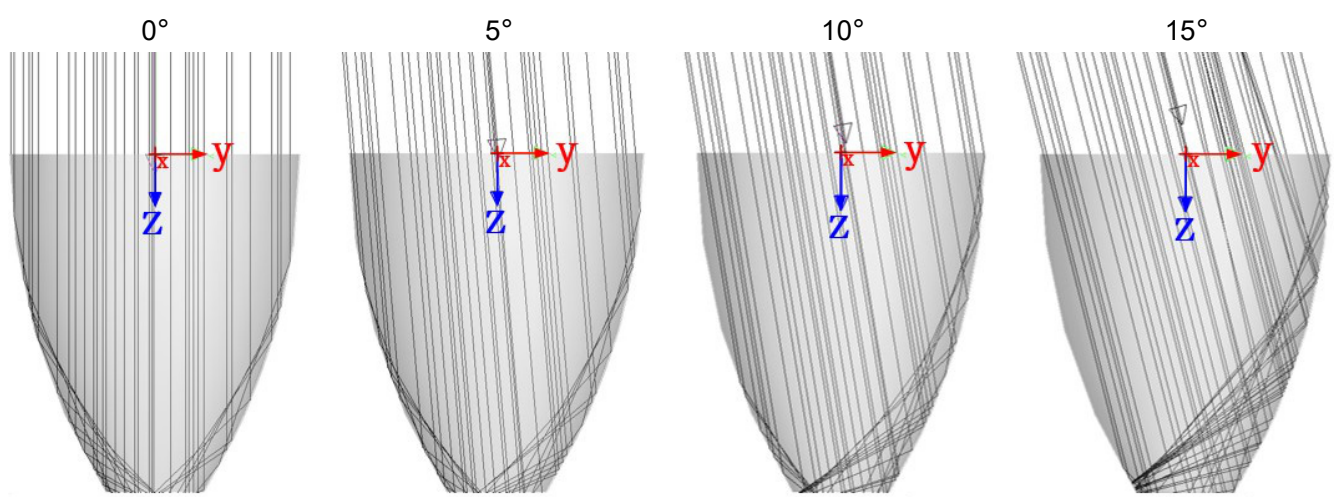

Fig. 3. The condensing effect of light when the incident angle is $0^{\circ}, 5^{\circ}, 10^{\circ}$, and $15^{\circ}$.

Assuming that the incident light is parallel light, theoretically, the maximum geometric condenser ratio (GCR) of CPC is

$$
C=\frac{1}{\sin \theta}
$$

In order to achieve the maximum GCR, $\theta=0$.

However, for the $\mathrm{CPC}$ of the certain parameter, the maximum condenser angle is

$$
\theta=\operatorname{asin}\left(\frac{2 f}{D_{\text {out }}}-1\right)
$$

Of course, the same CPC has different condensing effects for light of different incident angles. Figure 3 shows the concentrating situation at different angles. It is known that the maximum incident angle of CPC ( $\left.D_{\text {in }}=90 \mu \mathrm{m}, D_{\text {out }}=20 \mu \mathrm{m}, H=120 \mu \mathrm{m}\right)$ to achieve full concentrating is $12.8^{\circ}$. It can be found that the CPC incident angle is greater than $12.8^{\circ}$, and light is projected onto the wall of the cup and reflected.

However, in reality, the incident light may converge or diverge rather than parallel light. The value of $C$ is

$$
1<C<+\infty
$$

\section{Optical simulation}

We refer to the retinal structure of the elephant-nose fish and simulate the structure of the BII with Zemax software. The BII is a planar array consisting of $20 \times 20$ CPCs. The three-dimensional layout is shown in Fig. 4. The geometric parameters of the CPC in Fig. 4 are: $D_{\text {in }}=90 \mu \mathrm{m}, D_{\text {out }}=20 \mu \mathrm{m}, H=120 \mu \mathrm{m}$. (We choose this type of data as the initial data, and we will gradually optimize the parameters by considering the geometric concentration ratio and uniformity.) 
a

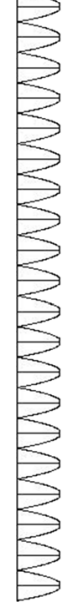

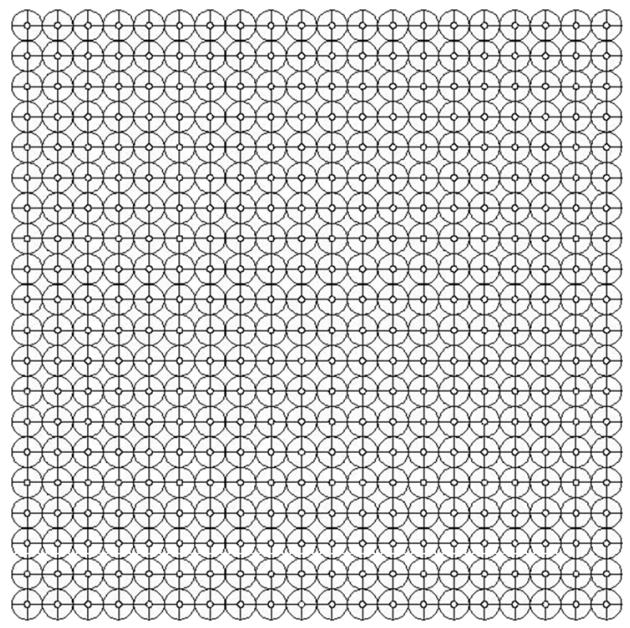

b

Fig. 4. The 3D layout of the BII. Side view (a), and front view (b).

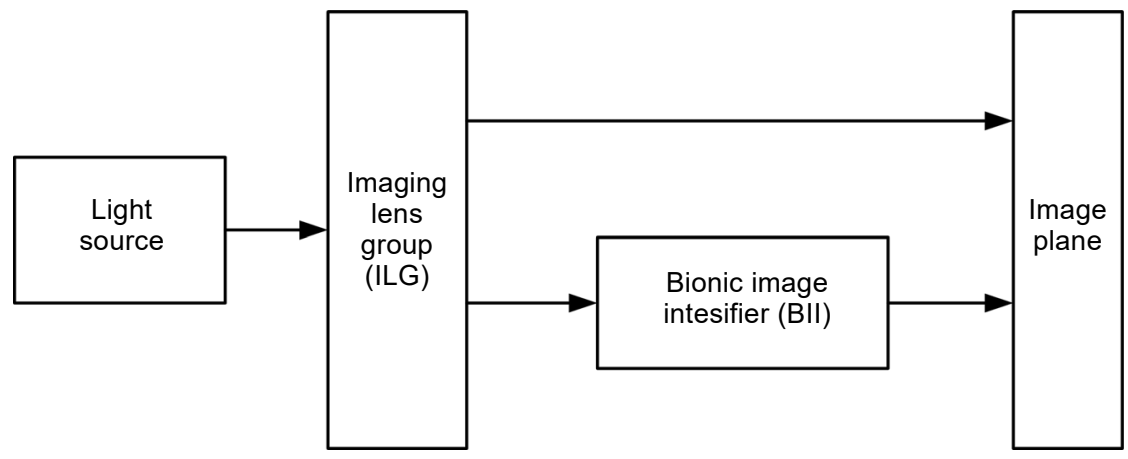

Fig. 5. The flowchart of simulation scheme.

In order to further understand the optical concentrating property of the BII, we conduct the simulation experiments. The simulation is divided into two groups. First group - the incident light passes through the ILG, and we observe the brightness of the image at the image plane. Second group - the incident light passes through the ILG and BII in sequence, and we observe the brightness of the image at the image plane. The flowchart is shown in Fig. 5. By comparing the simulation results of the two groups, we can understand the effect of BII in improving the imaging brightness. For observing the imaging system more intuitively, we have drawn schematic diagrams of imaging systems without BII and with BII, as shown in Figs. $6 \mathbf{a}$ and $6 \mathbf{b}$, respectively. Figure 6a shows an imaging lens and an imaging surface, and the imaging surface is on the focal plane of the imaging lens, with a focal length of $10.7 \mathrm{~mm}$. Figure $6 \mathbf{b}$ is based on Fig. $6 \mathbf{a}$, keeping the positions of the imaging lens and imaging surface constant, and placing the CPC array closely on the front surface of the image. The height of the CPC array 

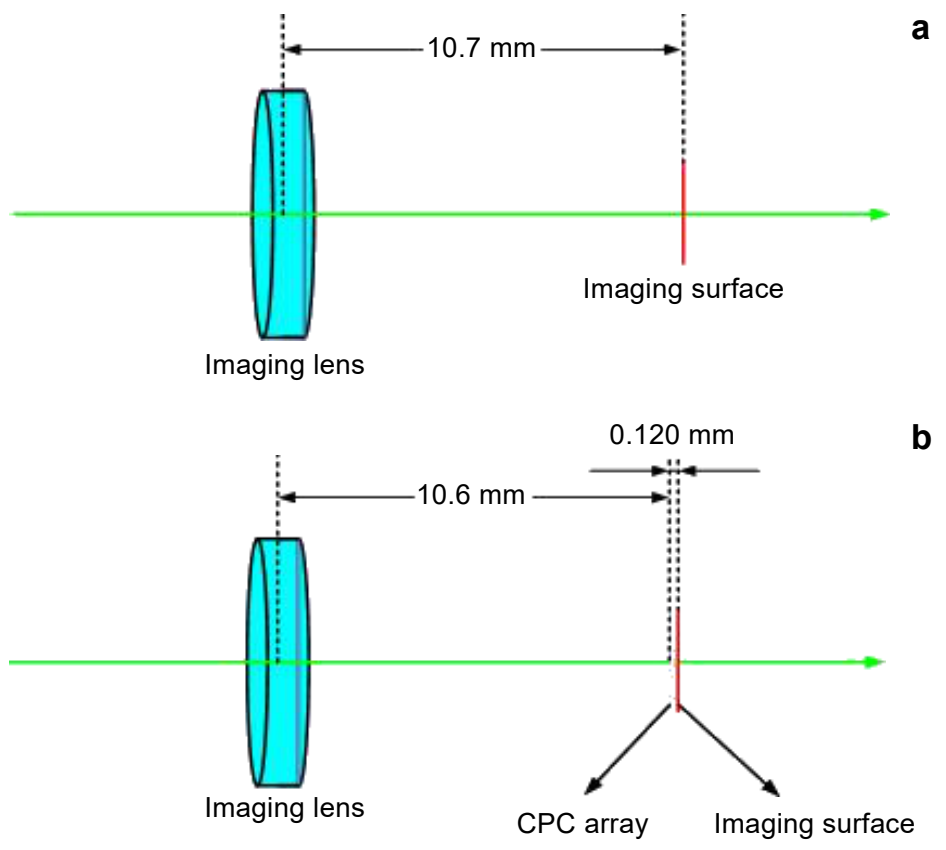

Fig. 6. The schematic of imaging system. Imaging system without BII (a), and imaging system with BII (b).

is $0.120 \mathrm{~mm}$, and the distance between the imaging lens and CPC array is $10.6 \mathrm{~mm}$, wherein, the image size is $2 \mathrm{~mm} \times 2 \mathrm{~mm}$. With the help of LightTools ray tracing software and Zemax imaging design software, we have concluded that the effective focal length of the imaging system is about $10.7 \mathrm{~mm}$.

The role of the ILG is imaging. The role of the BII is to increase the brightness of the image. They are independent of each other. In other words, the image quality depends on the ILG. However, it does not affect the geometric concentrating ratio of BII. Therefore, in order to simplify the simulation process, a simple single lens is selected for imaging using parallel light with a certain angle. The simulation diagram of Zemax is shown in Fig. 7. From this figure we can see two ray tracing states with BII and without BII.

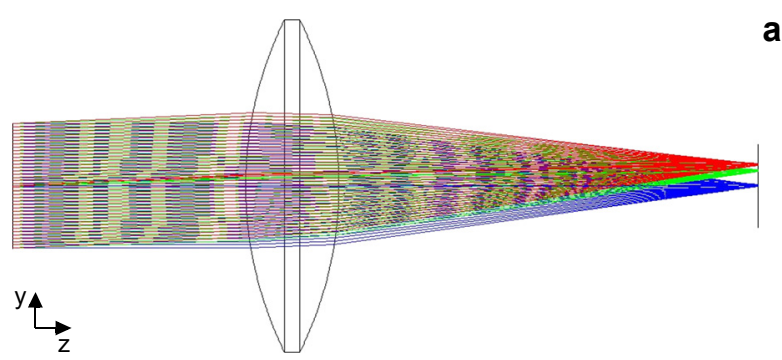

Fig. 7. The simulation diagram of Zemax. Vision system without BII (a), vision system with BII (b), and BII partial enlargement $(\mathbf{c})$. 


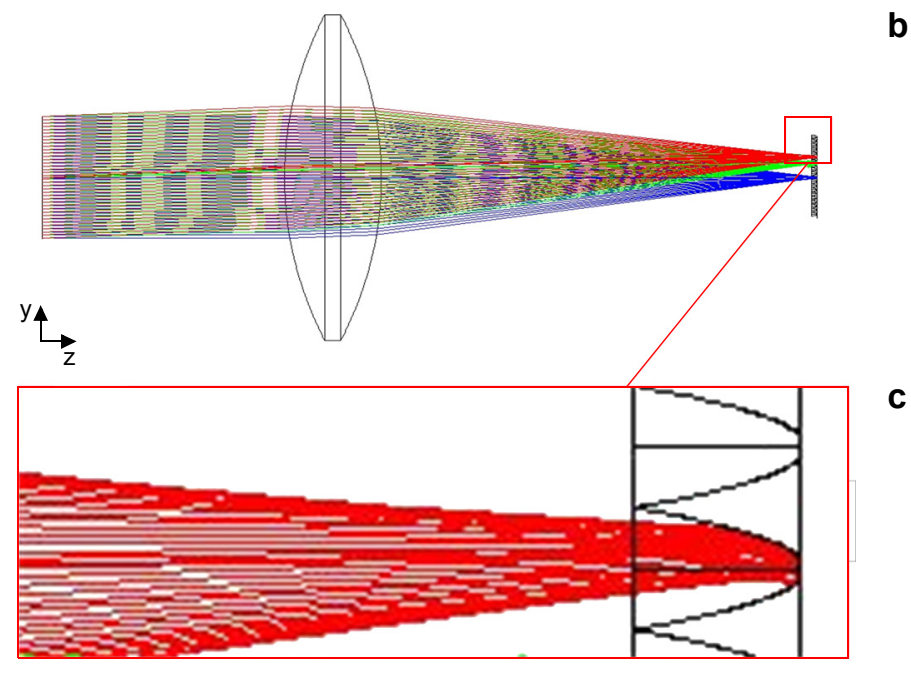

Fig. 7. Continued.

The geometric image analysis diagram is shown in Fig. 8. Figure 8a corresponds to the results of the first group of simulations, and Fig. $8 \mathbf{b}$ corresponds to the results of the second group of simulations. By comparing the labels on the right side, the BII improves the brightness of the image and has a certain concentration effect. However,
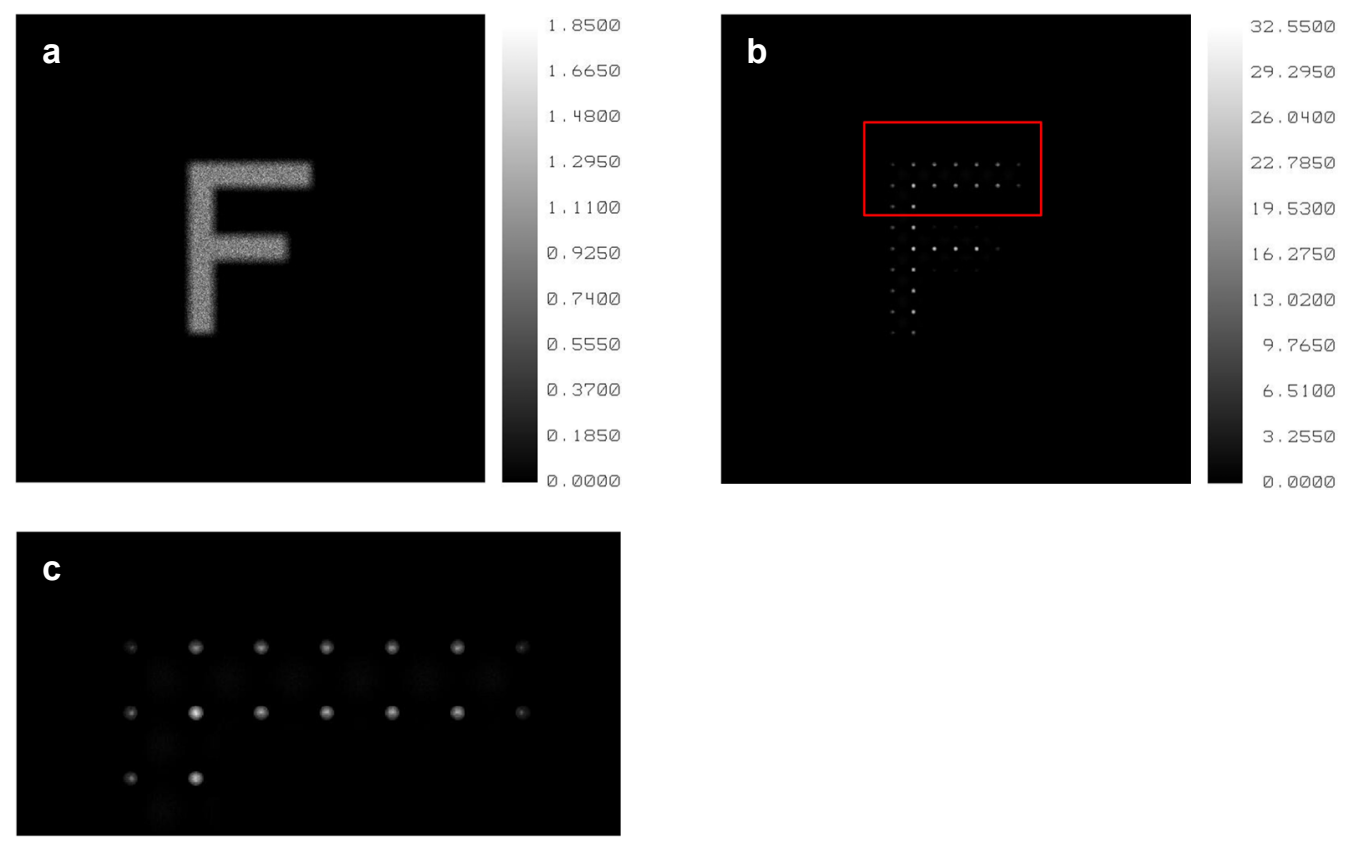

Fig. 8. Geometric image analysis diagram. Geometric image analysis diagram corresponding to Fig. 6a (a). Geometric image analysis diagram corresponding to Fig. $6 \mathbf{b}$ (b), and its partial enlargement (c). 
the quality of the image has dropped. This is because the diameters of the output port and the input port are inconsistent. We assume that the size of the original image in Fig. $8 \mathbf{a}$ is

$$
A=L \times W
$$

where $A$ is the area size of the image, $L$ is the length of the image, $W$ is the width of the image. However, because the BII is a CPC planar array, the BII divides the image into

$$
N=\frac{L}{D_{\text {in }}} \times \frac{W}{D_{\text {in }}}
$$

The image becomes discrete because of the loss of continuity, similar to the low resolution digital image. The light spot size is the area of the CPC output port.

Enlarge the part (red frame part) of Fig. 8b, as shown in Fig. 8c. It is found that concentration effect of some CPCs is not good when the light is oblique incidence. This affects the distribution of image points after brightness enhancement. We define the uniformity of the image as the distribution of image points after brightness enhancement.

In order to increase the accuracy of the simulation, the number of simulation rays is set to 25000 , and the final CPC parameters are used for analysis. The paper defines the light transmittance as the ratio of the number of rays received by the imaging surface to the total rays. Therefore, the transmission efficiency of light between $-12.8^{\circ}$ and $12.8^{\circ}$ is simulated, as shown in Fig. 9. It can be seen from this figure that the maximum transmission efficiency of light is $69.2 \%$ when the incident angle is $0^{\circ}$, and the minimum transmission efficiency of light is $54.6 \%$ when the incident angle is $\pm 12.8^{\circ}$. In order to be able to quantify the geometric concentration ratio of BII and the uniformity of the image, we construct a model similar to Fig. 7 in non-sequential mode. Parallel light is incident at different angles. In the condition of unit luminous flux, five

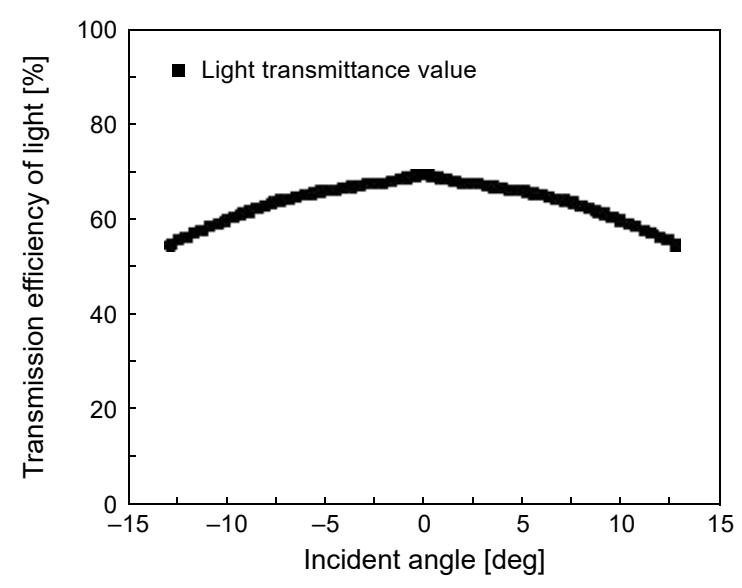

Fig. 9. The transmission efficiency of light from $-12.8^{\circ}$ to $12.8^{\circ}$. 


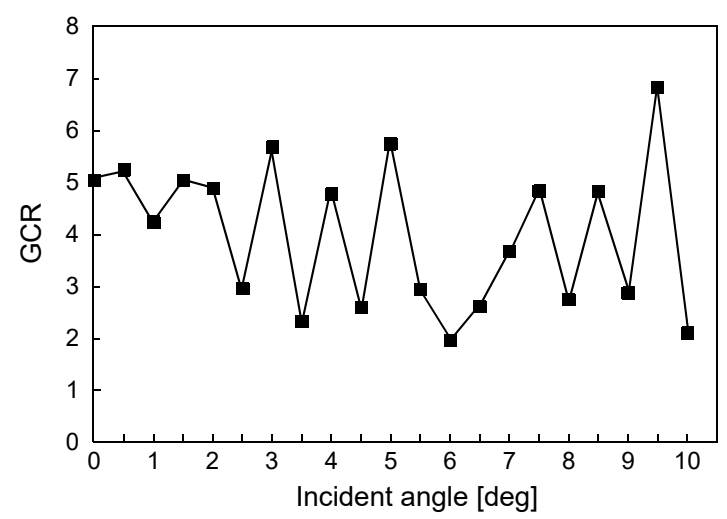

Fig. 10. The GCR of BII at different incident angles.

million rays are used for tracking simulation to analyze the illuminance distribution of planar detectors. The simulation results are shown in Fig. 10, where the GCR $C$ is related to incident angle $\varphi$, that is

$$
C=C(\varphi)
$$

From Fig. 10, we can see that the GCR of the CPC array floats up and down as the incident angle increases. The minimum is 1.95 and the maximum is 6.78 . The mean value is 3.97 and the variance is 1.41 . The value of GCR is not stable resulting from the effect of CPC for either diverging or converging light.

We define the expected value of the data as the GCR of the BII, and the expression is

$$
E(C)=\frac{1}{n} \sum_{i=1}^{n} C_{i}
$$

where $C_{i}$ is the GCR at different incident angles, $n$ is the number of data. In addition, we refer to the min-max normalization and standard deviation equation of the data to define the image uniformity, and the equations are

$$
\begin{aligned}
& C_{i}^{*}=\frac{C_{i}-C_{\min }}{C_{\max }-C_{\min }} \\
& D(C)=\left(1-\sqrt{\frac{1}{n-1} \sum_{i=1}^{n}\left(C_{i}^{*}-E\left(C_{i}^{*}\right)\right)^{2}}\right) \times 100 \%
\end{aligned}
$$

Equation (11) is the min-max normalization. Equation (12) is the standard deviation of the normalized data, and shows that the greater the value of $D(C)$, the better the uniformity of the image. After data processing, $E(C)=6.62, D(C)=72.39 \%$. 


\section{Structural optimization}

The simulation results validate that the BII model can improve the brightness of the image. We can be able to investigate the geometric concentrating ratio of the BII, the continuity and uniformity of the image dependence on the structure parameters of the CPC, for instance, the input port diameter $D_{\text {in }}$, the output port diameter $D_{\text {out }}$ and the height $H$. They are the key parameters in the actual design of CPC. They determine the geometry of the CPC and the performance of the BII.

\subsection{Image continuity}

The image continuity can be used as an evaluation criterion for structural optimization. The above simulation results (Fig. 8) show that the BII can make image lose the continuity. According to Eqs. (7) and (8), the image continuity is independent of the height $H$. All parameters in the image area $(A=L \times W)$ remain unchanged. We need to determine

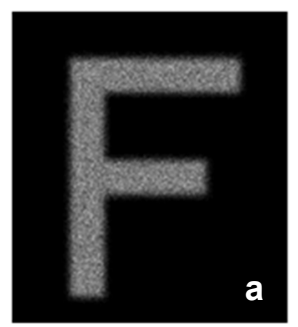

Without BII

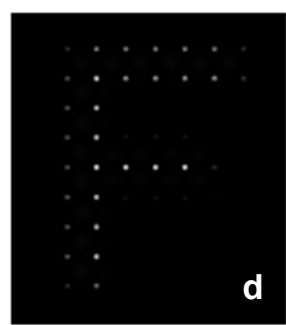

$90 \times 20 \times 120$

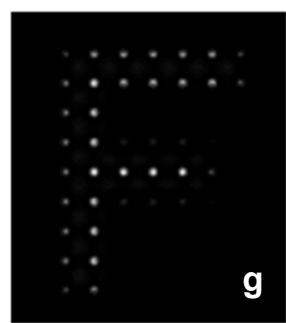

$90 \times 35 \times 120$

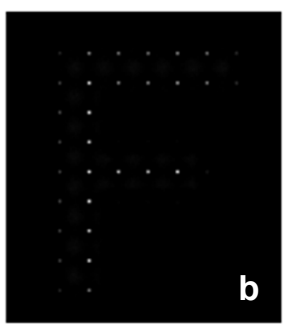

$90 \times 10 \times 120$

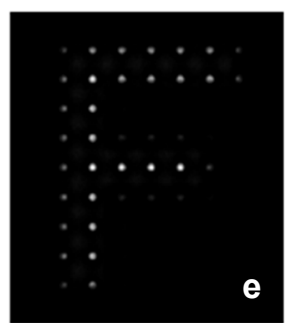

$90 \times 25 \times 120$

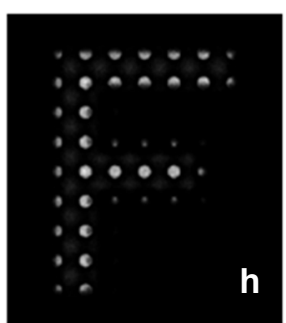

$90 \times 40 \times 120$

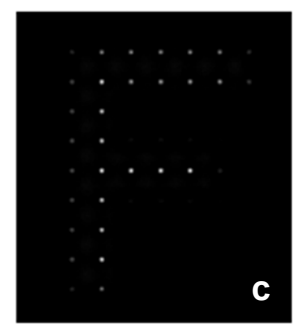

$90 \times 15 \times 120$

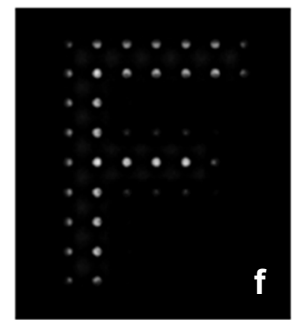

$90 \times 30 \times 120$

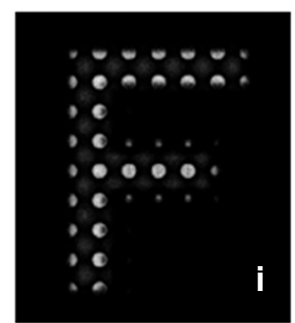

$90 \times 45 \times 120$

Fig. 11. The geometric image analysis diagram at different output port diameters. 
the effect of $D_{\text {in }}$ and $D_{\text {out }}$ on the image continuity. The simulation results of the relationship between image continuity and CPC structure parameters $\left(D_{\text {in }} \times D_{\text {out }} \times H\right)$ are shown in the figure.

Figure 11 shows the geometric image analysis at different output port diameters. The number of light spots is constant. The image continuity is independent of the output port diameter $D_{\text {out }}$, but $D_{\text {out }}$ determines the size of the light spot.

Figure 12 shows the geometric image analysis at different input port diameters. The image continuity is related to the input port diameter $D_{\text {in }}$. As $D_{\text {in }}$ gradually becomes smaller, the number of light spots will increase and the continuity will be better. It can be understood that the number of spots is similar to the pixels in the digital image. The higher the pixel, the better the resolution of the image. For example, the letter $F$ can be very intuitively recognized from Figs. $12 \mathbf{b}-12 \mathbf{e}$. When the value of $D_{\text {in }}$ increases, the information in Figs. 12k and $12 \mathbf{l}$ becomes blurred so that it cannot be accurately identified. However, the value of $D_{\text {in }}$ cannot be reduced indefinitely. On the one hand,

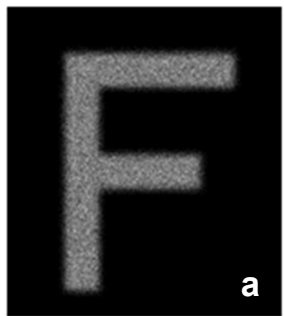

Without BII

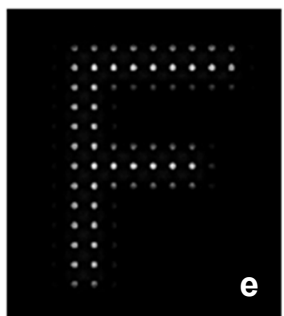

$60 \times 20 \times 120$

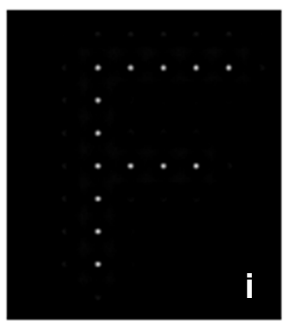

$100 \times 20 \times 120$

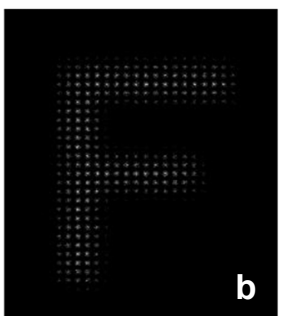

$30 \times 20 \times 120$

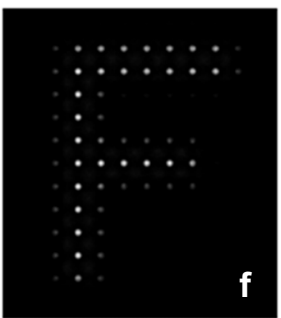

$70 \times 20 \times 120$

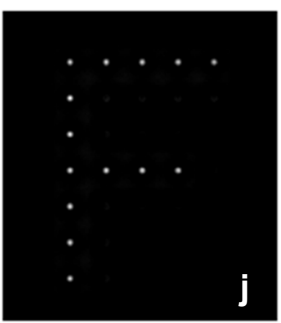

$110 \times 20 \times 120$

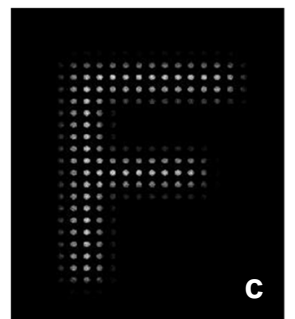

$40 \times 20 \times 120$

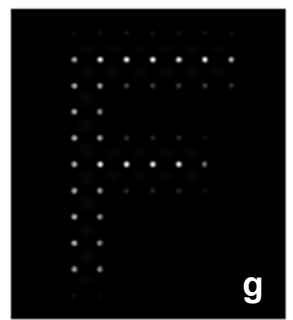

$80 \times 20 \times 120$

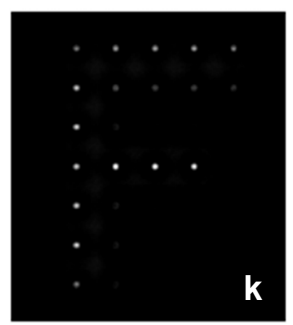

$120 \times 20 \times 120$

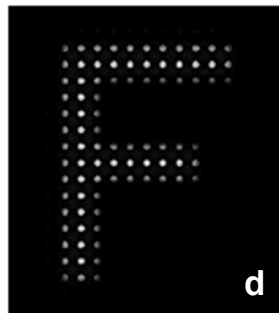

$50 \times 20 \times 120$

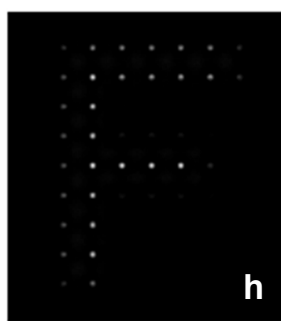

$90 \times 20 \times 120$

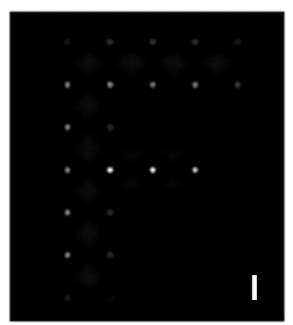

$130 \times 20 \times 120$

Fig. 12. The geometric image analysis diagram at different input port diameters. 
we compare Figs. 12b-12e. It is found that the concentration effect will be poor when the value of $D_{\text {in }}$ is reduced. On the other hand, it is the limitation of the processing technology.

In the simulation process, the value of $D_{\text {in }}$ is in the range of $30-130 \mu \mathrm{m}$. Therefore, as a result of comprehensive consideration, $D_{\text {in }}$ is $50 \mu \mathrm{m}$.

\subsection{GCR of BII and the image uniformity}

The image continuity is only related to the input port diameter $D_{\mathrm{in}}$. However, the GCR of the BII and the uniformity of the image depend on the structure parameters of the CPC, such as the input port diameter $D_{\text {in }}$, the output port diameter $D_{\text {out }}$ and the height $H$. Therefore, the image continuity is the first criterion to optimize $D_{\text {in }}$. The GCR of BII and the image uniformity are used as second evaluation criteria to optimize $D_{\text {out }}$ and $H$.

After determining the size of $D_{\text {in }}$, we need to determine $D_{\text {out }}$ and $H$. The simulation results of the relationship between the GCR of BII and the CPC structure parameters are shown in Fig. 13. When the GCR is maximum, $D_{\text {out }}=5 \mu \mathrm{m}, H=90 \mu \mathrm{m}$.

The simulation results of the relationship between image uniformity and CPC structure parameters are shown in Fig. 14. When the image uniformity is the best, $D_{\text {out }}=30 \mu \mathrm{m}$, $H=40 \mu \mathrm{m}$.

Through the analysis of Figs. 13 and 14, we find that as the height increases, the value of geometric concentration ratio gradually decreases, while the value of uniformity gradually increases, and finally both tend to be stable. This is because $D_{\text {in }}$ and $D_{\text {out }}$ are fixed, and the larger $H$ is, the smaller the curvature of the side wall of the $\mathrm{CPC}$ is. Meanwhile the amount of light entering is constant, the more uniform the spots are, and the smaller the concentration ratio is.

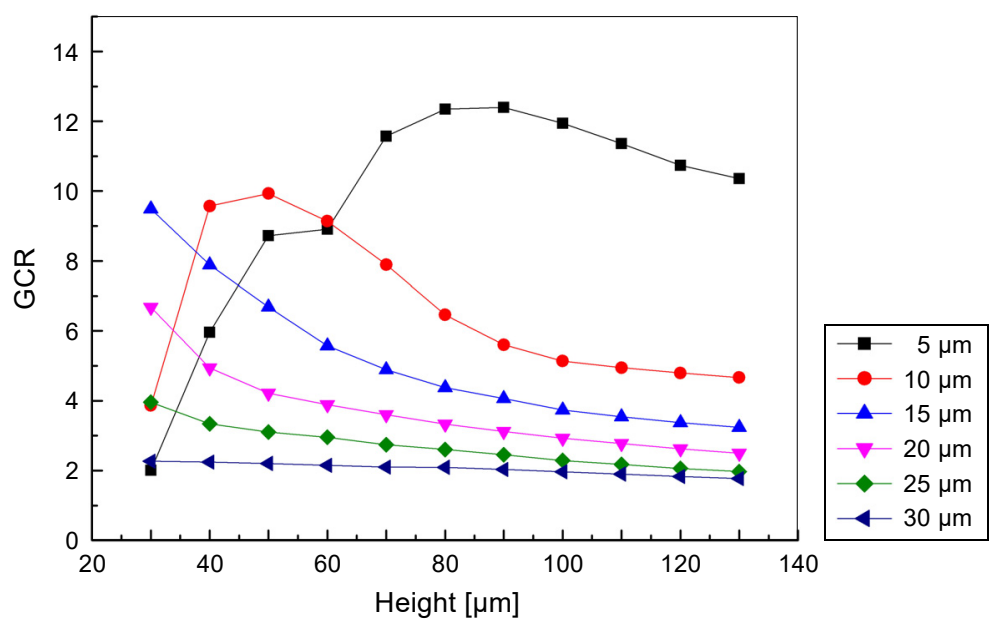

Fig. 13. Relationship between the GCR of BII and CPC structure parameters. 


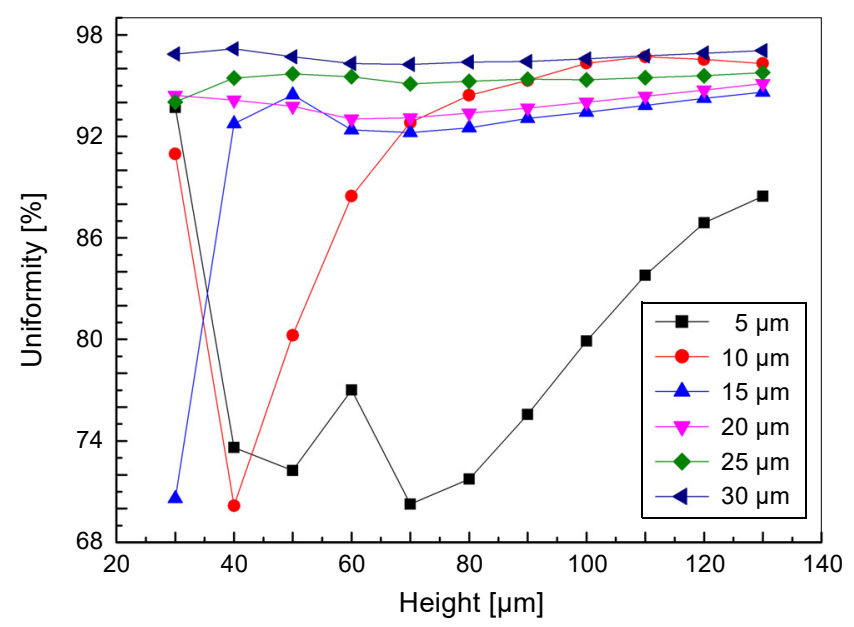

Fig. 14. Relationship between image uniformity and CPC structure parameters.

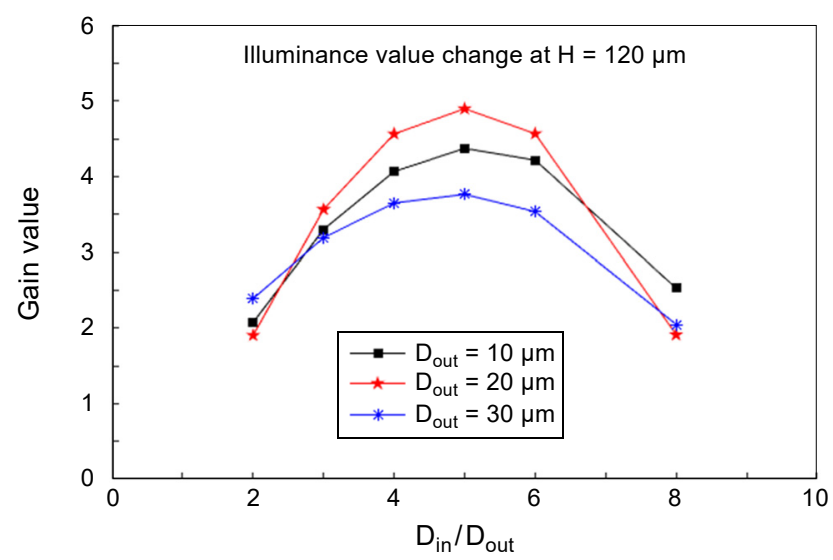

Fig. 15. Gain variation curve under different $D_{\text {in }} / D_{\text {out }}$.

When we keep the height $H$ of CPC constant ( $H=120 \mu \mathrm{m}$ as an example), as the ratio of input port diameter $D_{\text {in }}$ to output port diameter $D_{\text {out }}$ increases, the observed GCR of the CPC is shown in Fig. 15.

From the simulation results of the above figure, we find that the gain of CPC is the best when $D_{\text {in }} / D_{\text {out }}$ is about 5 .

From the curve change of Fig. 16, we can see that with the increase of CPC array $H$, the gain effect increases first and then decreases and finally stabilizes when $D_{\text {in }} / D_{\text {out }}$ $=5$. This is because the light concentrating effect is obvious in the early stage with the increase of CPC height. And when $H$ is gradually increased, the curvature of the CPC side wall is smaller, and the amount of light entering the CPC is constant, and the concentration ratio becomes smaller and finally becomes stable. 


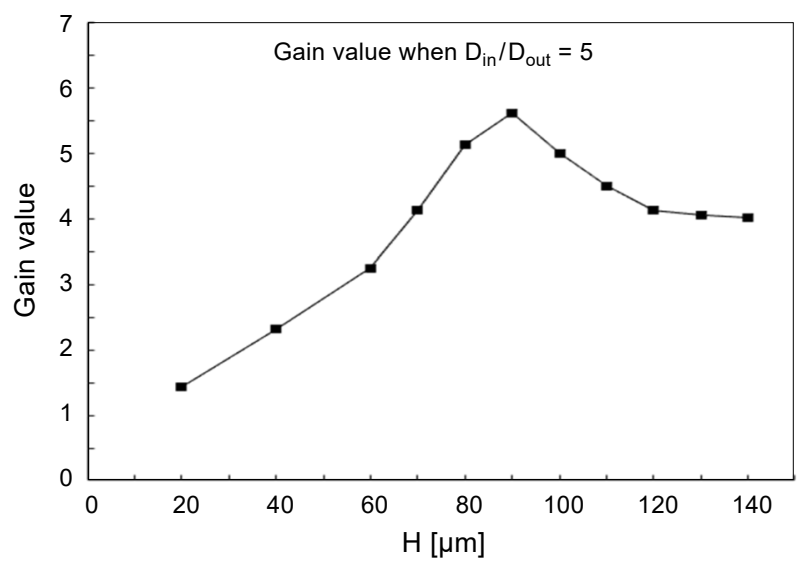

Fig. 16. Gain variation curve at $D_{\text {out }}=10 \mu \mathrm{m}$.

Considering the GCR of BII and the image uniformity, $D_{\text {out }}=10 \mu \mathrm{m}$ and $H=90 \mu \mathrm{m}$ are finally determined. The GCR is 5.61 and the image uniformity is $95.30 \%$.

When $D_{\text {in }}=50 \mu \mathrm{m}, D_{\text {out }}=10 \mu \mathrm{m}, H=90 \mu \mathrm{m}$, the simulation results reach the theoretical numerical optimum within a certain range.

\section{Discussions}

The elephant-nose fish has a unique retinal structure, so it has a strong night vision. Inspired by this kind of biological structure, the BII model composed of CPC planar array is established in this paper. The optical simulation results show the concentrating principle of the model. Subsequently, we used the image continuity, the GCR of BII, and the image uniformity as evaluation criteria to optimize the structural parameters of the CPC.

The bionic model in this paper has many advantages. (1) This model is a pure optical design, avoiding the use of complex electro-optical night vision technology and serious dependence on photoelectric materials; (2) The model has a good light-gathering ability, and the brightness can be increased by 5.61 times; (3) In the optimization process of the model, this paper gives a variety of evaluation criteria to ensure the rationality of the design; (4) The model adopts the planar array structure, which is conducive to manufacturing.

The bionic model in this paper has some limitations. (1) The image quality problems such as the image continuity and uniformity can be reduced to a certain degree, but cannot be eliminated; (2) The range of incidence angle in Fig. 10 is not fixed, it depends on the half of the FOV of the ILG; (3) The min-max normalization method is used to quantify the image uniformity. One drawback of this method is that when new data is added, it may cause changes in max and min, and it needs to be redefined; (4) The smaller size of the CPC makes processing more difficult and costly.

In addition to the limitations, there are still many deficiencies that require further study in this paper. (1) The current relevant conclusions are given on the basis of sim- 
ulation, and we need to improve the experimental work; (2) This model is different from the principle of electro-optical night vision technology, but it does not exclude it. In the future, we can strengthen the research on the compatibility between this model and electro-optical technology, and can further improve the night vision technology.

\section{Conclusion}

In summary, we simulated a BII based on the unique retina structure of the elephant -nose fish, achieving the goal of improving image brightness in low light conditions. The BII is a planar array designed according to the principle of CPC concentration. The process of determining CPC structural parameters in consideration of BII's GCR and imaging effects is described in detail. The simulation results show that the BII structure has good GCR and image uniformity. The final optimized structural parameters are: input port diameter is $50 \mu \mathrm{m}$, output port diameter is $10 \mu \mathrm{m}$, and the height is $90 \mu \mathrm{m}$. The comprehensive performance of BII is optimal. The geometric concentration ratio is 5.61 . The image uniformity is $95.30 \%$. The work in this paper shows that the bionic image intensifier can improve the brightness of the image and enhance the visual ability in a low illumination environment.

Acknowledgements - This work was supported by National Natural Science Foundation of China (NSFC) (61675153).

\section{References}

[1] KоLв H., How the retina works: much of the construction of an image takes place in the retina itself through the use of specialized neural circuits, American Scientist 91(1), 2003, pp. 28-35.

[2] Morris N.J., Night walking: darkness and sensory perception in a night-time landscape installation, Cultural Geographies 18(3), 2011, pp. 315-342, DOI: 10.1177/1474474011410277.

[3] TAO Y., Jin W.-Q., WANG Y., SHI F., GuO H., The MTF analysis of high performance proximity image intensifier, Guangzi Xuebao/Acta Photonica Sinica 45(6), 2016, article 0604003, DOI: $10.3788 /$ gzxb20164506.0604003.

[4] Li J.P., Zhang Y., Wang Y., The current situation and development of low-light-level image intensifier and its core materials, Guangxue Jishu/Optical Technique 43(3), 2017, pp. 284-288.

[5] BAI X.F., YANG S.N., Hou Z.P., NVIS compatible characteristic of low light level image intensifier assembly, Infrared and Laser Engineering 45(S2), 2016, pp. 101-105.

[6] Ni Y.R., Lu C.H., Meng X.F., Xu Z.Z., Zhang Q.T., Novel filter materials for glimmer detector, Journal of Nanjing University 25(4), 2003, pp. 69-72.

[7] Ni Y.R., Lu C.H., Meng X.F., Xu Z.Z., A study of a new type of light absorbable glimmer detector, Materials Review 17(7), 2003, pp. 73-75.

[8] Liu H., Huang Y., Jiang H., Artificial eye for scotopic vision with bioinspired all-optical photosensitivity enhancer, Proceedings of the National Academy of Sciences of the United States of America, PNAS 113(15), 2016, pp. 3982-3985, DOI: 10.1073/pnas.1517953113.

[9] Lopez-Sanchez O., LembKe D., Kayci M., Radenovic A., Kis A., Ultrasensitive photodetectors based on monolayer $\mathrm{MoS}_{2}$, Nature Nanotechnology 8(7), 2013, pp. 497-501, DOI: 10.1038/nnano. 2013.100.

[10] Liu C.H., Chang Y.C., Norris T.B., Zhong Z., Graphene photodetectors with ultra-broadband and high responsivity at room temperature, Nature Nanotechnology 9(4), 2014, pp. 273-278, DOI: 10.1038/nnano.2014.31. 
[11] Vashchenko V.A., Sinkevitch V.F., Physical Limitations of Semiconductor Devices, Springer US, 2008, DOI: 10.1007/978-0-387-74514-5.

[12] SoRenSEn R., We see in the dark, Noûs 38(3), 2004, pp. 456-480, DOI: 10.1111/j.0029-4624.2004. 00478.x.

[13] Warrant E.J., Johnsen S., Vision and the light environment, Current Biology 23(22), 2013, pp. R990 -R994, DOI: 10.1016/j.cub.2013.10.019.

[14] Francke M., Kreysing M., Mack A., Engelmann J., Karl A., Makarov F., Guck J., Kolle M., Wolburg H., Pusch R., der Emde G., Schuster S., Wagner H.-J., Reichenbach A., Grouped retinae and tapetal cups in some Teleostian fish: occurrence, structure, and function, Progress in Retinal and Eye Research 38, 2014, pp. 43-69, DOI: 10.1016/j.preteyeres.2013.10.001.

[15] Kreysing M., Pusch R., Haverkate D., Landsberger M., Engelmann J., Ruiter J., Mora-Ferrer C., Ulbricht E., Grosche J., Franze K., Streif S., Schumacher S., Makarov F., Kacza J., Guck J., Wolburg H., Bowmaker J.K., von der Emde G., Schuster S., Wagner H.-J., Reichenbach A., FRANCKE M., Photonic crystal light collectors in fish retina improve vision in turbid water, Science 336(6089), 2012, pp. 1700-1703, DOI: 10.1126/science.1218072.

[16] Landsberger M., von der Emde G., Haverkate D., Schuster S., Gentsch J., Ulbricht E., Reichenbach A., Makarov F., Wagner H.-J., Dim light vision - morphological and functional adaptations of the eye of the mormyrid fish, Gnathonemus petersii, Journal of Physiology-Paris 102(4-6), 2008, pp. 291-303, DOI: 10.1016/j.jphysparis.2008.10.015.

[17] Zhang H., Chen H., Han Y., Liu H., Li M., Experimental and simulation studies on a novel compound parabolic concentrator, Renewable Energy 113, 2017, pp. 784-794, DOI: 10.1016/j.renene.2017.06.044.

[18] Su Z., Gu S., VAfai K., Modeling and simulation of ray tracing for compound parabolic thermal solar collector, International Communications in Heat and Mass Transfer 87, 2017, pp. 169-174, DOI: $10.1016 /$ j.icheatmasstransfer.2017.06.021.

[19] JaAz A.H., Hasan H.A., Sopian K., Mohd Hafidz Bin Haji Ruslan, Saleem Hussain Zaidi, Design and development of compound parabolic concentrating for photovoltaic solar collector: review, Renewable and Sustainable Energy Reviews 76, 2017, pp. 1108-1121, DOI: 10.1016/j.rser.2017.03.127. 